\title{
Public Service Motivation and Public Sector Incentive
}

\author{
Lamei Tang ${ }^{1, a}$ \\ Southwest Jiaotong University, Cheng Du,Sichuan, 610036 \\ ${ }^{\mathrm{a}}$ email,
}

Keywords: PSM, Public Sector Incentive Economic Incentive Theory, Principal-Agent Theory

\begin{abstract}
In this paper we elaborate the reason why public sector incentives need to take a full acknowledgment and consideration about the organizational publicity and public servants' psychological motivation.and we also try to demonstrate the implementation logic of public sector incentives from the personal motivation,organizational characteristic and the relationship between incentive and job performance,by reviewing the basic assumptions and empirical research results, which are mainly about public service motivation (PSM) theory applied to the public sector incentive.Finally, under the PSM theory,we put further consideration about public sector incentives:Measurement optimization for PSM, intrinsic incentive internalization system design and causal analysis between PSM and incentive performance. Public sector incentive issue is one of the core issue of public human resource management.Comparedwith the private sector, public sector has issuesin implementing incentive ,such as organizational goal ambiguity target diversification and hard budget constraint, because of its publicity.Under these issues,public sectorincentive shows two features:the extrinsic incentive is limited and the intrinsic incentive issignificant.But,for a long time, public sector use the private sector's economic incentive model directly.So it assumesthat public servants are"rational economic participant ",and promotes the substance, promotion, competition, etc in the incentives.But this ignores the public spirit of organization and the influence of organization system and culture.Itdoesn't change the situation of administrative inefficiency and lack of vitality.Since present research lacks reference carding about the logical description of PSM theory applied to public sector incentive,we want to explore that in this paper.So, firstly we have critical thinking about current economic incentive theory applied to the public sector ,then comb the logical relation of incentive object(servant motivation),subject (public organization) and goal(organizational performance).In the end of this paper, we have been thinking something newabout PSM theory applied to public sector incentive.
\end{abstract}

\section{Critical Reflection of Traditional Economic Motivation Theory}

For a long time,public sector use the traditional economic motivation theory,so it emphasizes to adopt salary, promotion and competition to form a set of incentive system for public servant,which is easy to institutionalize and quantify in theory.Its main theoretical basis is "Principal-agent Theory",which propose the agent and client are both "rational economic participant"and have the issue of inconsistent interests and asymmetric information.Therefore, there is supervision cost, and it is better to establish an incentive system based on agent performance.In the risk sharing and incentive compatible way,it can motivate agents to improve efficiency.this kind of incentive need two preconditions:firstly,the client needs to give the agent a definite working objectives.Secondly,the performance of the agent is contractual(the agent performance is capable of being observed and verified by third parties).[1]But for public sector,this incentive theory has following problems:

Firstly,inadequate consideration of the organizational context of the public sector.Moe(1984)said that public sector incentive has many particularities.it shouldn't motivate public servant by using the economic incentive theory directly. Or it would cause serious misleading.Tirole(1994)proposed 
that economic incentive theory is only adapted to private sector considering the organizational characteristic.[2]Specifically, the public sector is different from the private sector in terms of organizational goals, ways of operation, values, and organizational governance structures, which lead the seven special problems for public sector incentive:(1) the amount of output and quality is difficult to measure (2) the principal-agent relationship of multiple complex (3) the lack of a clear, unified goal (4) multi agent task Officer (5) public property has inherent exclusivity (6) limited official relative performance (7) existing public hard budget constraints the public sector.These objective organizational contexts show that there are two dilemmas for the implementation of economic incentives in the public sector.First, the objective budget is hard to restrain, and the political clients are restricted to provide sufficient material incentives for public officials.Second, there are many challenges in the incentive process, and it can even cause distortion of incentive (the final effect of incentive mechanism to guide the agent's behavior deviates from the client's expected goal).About that, the principal-agent analyst and political scientist Miller and Whitford believe the key to solve the dilemma is to find a more suitable motivation explanation for the public servant and suitable way for public sector incentives.[3]Moreover, many scholars have found that the public sector has the phenomenon that agents are proud of their work and make efforts for it,which can not be explained by economic intensive theory.they once said public sector is suited to intrinsic incentives,but no further explanation or analysis are given to the reasons behind such phenomena.

Traditional economic incentive theory attaches great importance to the allocation of resources. It concerns the efficiency management within the enterprise, and lacks attention to human needs and behavior.It is the basic reason that the hypothesis of "rational economic participant"can not explain the psychological motivation of the public servant.They think both the client and the agent are purely selfish individuals, so they encourage material incentives , but series of problems haven't be fundamentally improved to public servant, such as lazy workattitude, low efficiency, rigid image, low creative ability and poor performance.Ma Ling(2003)put forward from the view of psychological contract that the psychological development level of modern organization members is increasing day by day, and the existing economic contract can't transfer and exert the enthusiasm and creativity of organization members to the maximum extent.[4]

Through the above research results,we find there is an essential difference of incentives between the public and private sectors.and the understanding of organizational context and individual motivation have become particularly important. What is the logical relationship of the organizational characteristics of public sector,the motivation behavior of public service personnel and public sector incentives? In recent years, the new motivation theory closely related to the public sector "the theory of PSM” has given us new thinking.

\section{The Theoretical Basis and Hypothesis of Organizational Motivation Based on PSM Theory}

Public service motivation was formally proposed by American scholars Perry and wise in 1990, which in European studies are often referred to as the spirit of public service and the ethics of public service.By reviewing the public choice theory and the new public management theory applied to the public sector,this theory discard the first two theories' propositionthat the public service servants are"rational economic participant"and all the departments need use a lot of external incentives, such as money, promotion, competition system to manage and train their employees.As an intrinsic psychological process, public service motivation is a multi-dimensional and abstract concept, so it is difficult to define and operate. Scholars have proposed many different forms but compatible concepts.One of the more authoritative definition came from Perry and Wise, who defined PSM as 
"the tendency of individuals, primarily or uniquely, to be driven by public institutions and organizations." and emphasis on that civic responsibility and compassion is relevant to public organizations.they also gave the three basic assumptions of PSM:Proposition 1, The greater an individual's public service motivation, the more likely the individual will seek membership in a public organization;Proposition 2, In public organizations, PSM is positively related to individual performance;Proposition 3,Public organizations that attract members with high levels of public service motivation are likely to be less dependent on utilitarian incentives to manage individual performance effectively.[5]These three propositions lay the foundation for the study of related topics in the following researches.

The basic hypothesis of PSM and organizational motivation originally came from the study of Knoke and Wright-Isak's predisposition-Opportunity model.The model defines motivation as "a tendency to manifest itself under certain external cues."In this model eight different organizational incentive system typologies are created, including pure utility and service incentive systems.Perry and Wise think if PSM represents a tendency can be seen, the incentives (i.e. external cues) is provided the motivation for its members to express commitments, and that is the opportunity side of the model.Subsequent studies have shown that this view does not deny public employees' concerns about physical incentives or external compensation,but only emphasizes the degree of dependence on the physical and money of the public organizations when stimulating individual performance.[6]This provides the public sector with an incentive perspective for sustainable development at the economic and motivational levels.

\section{Empirical Studies of PSM and Public Sector Incentives}

Empirical studies of PSM and public sector incentives are focused on the following three aspects:public servant PSM, argumentation and measurement,analysis of public sector organizational situations and correlation analysis between individual motivation and incentive performance.From an incentive point of view, it is mainly based on the PSM theory of motivation to do object motivation analysis, subject organization, situational thinking and incentive target demonstration.

Analysis of Motivation Based on Incentive Object. Basing on the PSM theory, scholars have been demonstrating and measuring the motivation of public service workers.Studies show that public service participants are ubiquitous in PSM.So,they have a spirit of public service that is willing to sacrifice and sacrifice, that is, altruistic motivation, and generally concerned about the interests of others more than their own interests.they also may feel satisfied with the work itselfeven without paying.It reflects the effectiveness of intrinsic incentive for public service workers, and it finds new way for organizational incentive in the public sector.

This has led scholars to study the reward preferences of employees in public and private sector organizations.Most studies have shown that public sector employees prefer non-material incentives to private sector employees.Crewson(1997) finds out that public and private sector employees do not have significantly different preferences for high wage pay through surveys of wage preferences for public and private sector employees.But relative to other external rewards, public sector employees value intrinsic rewards more, relying less on utilitarian incentives.Rainey(1982) a survey of 275 middle and lower levels of public and private sector managers found that public sector employees did not see economic compensation as important as the private sector.[10]Houston(2000) in the study of public and private sector preferences, employees in the public sector have also paid more attention to the sense of work and achievement;Wright(2007) also suggest that intrinsic rewards may be more important than performance compared with external rewards in the public 
sector,after giving work motivation, team mission and external rewards assessments for 807 state representatives in New York.[7]

Some scholars have further explored the influence of intrinsic and external incentive on the basis of Motivation Crowding Theory.Francois and Vlassopoulos (2008) argue that external incentives have crowding out effects on PSM performance.But Georgellis thought (2011) this crowding out effect occurs only in a few public sectors, such as the health and high-education sectors.In the follow-up research, some scholars have suggested that proper external motivation can achieve the crowding-in-effects for both.Taylor and Weiss Waugh conduct a questionnaire survey about public sector staffs from the United States, Canada, Britain, Germany, France, Denmark and Norway. They found intrinsic incentive is the main antecedent variable to achieve external incentives in the public sector.[8]Although there are no consistent results, the research point of view is that the internalization of external compensation and the strengthening of intrinsic compensation are the preconditions for effective public sector incentive.

Demonstration of the Public Sector Organization Context. The economic incentive theory has blurred the distinction and definition between public and private organizations.Economic incentive theory propose public servant as"rational economical participant", providing a large number of external incentives, but inevitably lead to inappropriate incentives.Di Giulio pointed out that the reason why there would be improper incentives is that the principal-agent model can explain some bureaucrats'lazy, dereliction of duty and even abuse of power behavior.But this cannot explain the dedication, compassion and even sacrifice silently".He believes that this is because there is a cultural basis for bureaucratic behavior, that is, their behavior will be affected by social and ethical factors,Moreover, organizational leaders can achieve such public organizational characteristics by creating and maintaining the organizational culture of "principled agents".Perry put forward a similar view that the prosocial behavior of public organizations means that the analysis of the motives of bureaucratic behavior should "bring society in."we should take full consideration about that individual values and preferences is acquired in the social process.[9]This is the important content of PSM theory related to organizational administration culture and system.

Based on the above objective characteristics of public organizations, PSM theory holds that public sector incentives exist objective environment conditions for intrinsic incentive,Correspondingly to the person-organization fit theory (POF) .Based on this theory, scholars believe that the public sector organizations can attract and train members with high PSM through the process of "attraction, selection and absorption".This process has been widely used in the recruitment and management of human resources.For example, individual and organizations attract, select each other, and then organizations create a good atmosphere of the organization (including organizational culture and system) to strengthen the matching between each other.The public sector thus has the foundation for the implementation of intrinsic incentives. Vandenabeele also have also found that the government and other public sectors have a special attraction for people with high PSM.[10]

Analysis and Demonstration of PSM and Public Sector Incentive Objectives. The traditional motivation theory tells us that through the development and function of individual motivation, we can directly influence individual's work attitude, and finally lead individuals to achieve job performance.For organizations, the ultimate goal of motivation is performance.But as we mentioned above, modern organization members pay more and more attention to their physical and mental development, personal show is not concerned about their own performance, but in the public organization of such specific organization environment, role conflict will be weakened, more property prepared a social person.Therefore, the performance of individuals and public 
organizations is the key goal of public sector motivation, that is, to achieve the coordinated development of individuals and organizations. The current empirical analysis are mainly about the correlation of the PSM, work attitude and job performance (including organizational and personal performance).from that we can see the way the way of PSM impact on job performance(Figure 1)

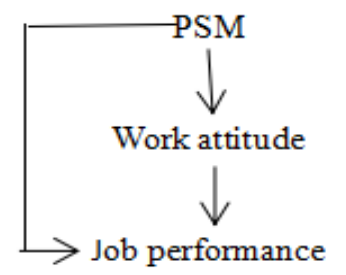

Figure 1:The influence approach of PSM to job performance

First of all, the research on the correlation between PSM and work attitude,.Theoretical and empirical research shows that the two have direct and indirect relations in different dimensions and levels.The diversity and complexity of human beings determine the diversity of their subjective work attitude.People oriented motivation pays more and more attention to the attitude of employees because it is directly related to the performance of individuals and organizations.Rainey(1982) initially combines work attitude with PSM theory.He used direct questioning to investigate the attitude of public and private sector employees' pay preference, and found that public service motivation had an important positive impact on the attitude of civil servants.Crewson and Houston give further explanation of the reason why personal PSM have a positive influence on the work attitude and work preference,by operationalizing the PSM as the difference between personal service orientation and personal economic orientation.[11]Some scholars have analyzed correlation about dimensions of work attitude scale, such as job satisfaction, job involvement, values, organizational citizenship behavior and organizational commitment with PSM.Among them, the dimension of job satisfaction is more concerned.Scholars such as Naff,Crum((1999))and Kim (2004) have find that the motivation of public service has a significant impact on job satisfaction.[12]The dimensions of job involvement, scholars and Buchanan (1975) Rainey (1982) do research about job involvement in the private sector and the public sector through analysis of public service motivation variables.The conclusion is that public sector investment is lower than private sector, and is weakly related to public service motivation (or behavior).[13]Scholars like Zhu Jun(2013)have done an empirical research on young civil servants based research of Buchanan.he found that the two dimensions attractiveness of public policy making and self dedication of PSM have a significant impact on job involvement.[14]Liu Xin used the job satisfaction as the dependent variable, public service motivation as a mediating variable, by a direct empirical measurements of 684 civil servants of China central ministries to local provinces.He found PSM have a direct influence on the civil servants' job satisfaction.[15]Some scholars also refine the five dimensions of the public service motivation scale, and analyze the correlation between the five dimensions and job satisfaction one by one.

Secondly, the empirical analysis between PSM and job performance.Perry and Wise (1990)proposed there is a positive correlation between public service motivation and job performance.Alonso and Lewis (2001) examined the public service motivation and job performance of 35000 federal white-collar civil servants by using multiple regression and logical analysis .they found that public service motivation has a significant correlation with performance score and performance level.Through empirical research and analysis on the Chinese MPA graduate first hand investigation ,Li Xiaohua, Dong Jun (2012) confirmed that the public service motivation has a 
positive effect on individual performance.In other words,thehigher performance the civil servants with PSM have ,the higher of their interpersonal promotion, job dedication, task performance.[16]Although the early study of Rainey and Steinbauer had proposed that public service motivation is positively related to organizational performance rather than job performance.[17]But Ruiz in the survey of 13532civil servants in Swiss Confederation discovered there is no significant relationship between the attractiveness of public policy making and organizational performance.[18]Although the initial study of the relationship between PSM and job performance aroused argument. Whether there is a significant relationship between the two, and is it positive or negative?There was no consistent confirmation.However, most studies show that there is a certain degree of association between PSM and job performance.

\section{Conclusion}

According to the review PSM theory and public sector incentive theory .We explore the relationship of public organization and individual performance and characteristics of PSM ,basing onthe public service motivation theory.We find the theoretical and empirical research on the PSM and the public sector incentives need to be strengthened from the following aspects:

First,we should use the measurement according with modern psychometric methods to determine and measure the implicit motive. One of the biggest challenge of PSM theory is how to optimize the measurement dimensions.It needs to overcome the "preference falsification","social expectation effect" and other endogenous factors of psychological variables .Greenwald (1998) put forwarda new research method of implicit social cognition-- impact Association test (IAT)method.It is a computerized discrimination task with reaction time as index. We can measure the implicit attitude of an individual indirectly through the evaluation of the automatic connection between conceptual words and attribute words.[19If this method is effectively combined with the PSM measurement, the persuasiveness of PSM can be enhancedtheoretically.Andin the future, it will provide an important reference for the selection and promotion of talents in the public sector..

Second,we need to do further research about the incentive internalization system design and practice application.Study on PSM theory and public service demonstrates the feasibility of incentive incentive internalization in the public sector.But there is a lack of system operation design and practical application of research and analysis. The incentive is still the internalization of idealism , which can not be copied.

Third, till now we don't find enough research about the causal relationship between the work performance and PSM .The key way to evaluate an incentive method is to quantify the incentive effect.But through the survey,we findexistingempirical studiesbetween PSM and work performance are mainly about theircorrelationstudy.those studies prove whether the two are related ,but there is no causal analysis in depth .In addition, if PSM do have impact onjob performance, So how much will it affect? And this will be the key way to quantify the effect of PSM on incentives.it will have great significance to the study of public sector motivation.

\section{Reference}

[1][3]HuangZaisheng,Analysis of organizational motivation theory in public sector[J].Foreign economy and managemen.2015(01).

[2]Tirole, Jean .The internal organization of governmen t[ J] . Oxford Economic Papers, 1994 , 46 : $1-29$. 
[4]Ma Ling. The breakthrough point of modern organizational motivation -- psychological contract[J].Department of management abstracts, 2003, (03): 59-61.

[5]Li Yubao, $\mathrm{Yu}$ tianqi.Organizational motivation, literature review[J].Industrial technology and economy, 2005, (02): 93-94+97.

[6][8]JL Perry ,A Hondeghem ,LR Wise.Revisiting the Motivational Bases of Public Service:Twenty Years of.

Research and an Agenda for the Future[J].Public Administration Review.2010 , 70 (5) :681-690.

[7]Zhang Suhong, Kong Fanbin.Shaping the spirit of public service from the perspective of public service motivation[J]. Nanjing Social Sciences, 2016, (11): 75-81.

[9]Zeng Junrong.Public service motivation: concept, characteristics and measurement[J].Administration of China, 2008, (02): 21-24.

[10]W Vandenabeele.Government Calling: Public Service Motivation as an Element in Selecting Government as an Employer of Choice[J].Public Administration,2008,86(4):1089-1105.

[11]Li Xiaohua,The structure and measurement of public service motivation[J].Journal of Wuhan University (PHILOSOPHY AND SOCIAL SCIENCE EDITION)2008,(06):797-802.

[12]Kim .individual-level factors and organizational performance in government organizations [J].journal of public administration research and theory .2005,15:245-261.

[13]Kanungo,R.measurement of job and work involvement[J]. journal of applied psychology.1982,67(3):341-349.

[14]Zhu Jun.The impact of public service motivation on job involvement of young civil servants[J].journal of China Youth Political college,2013,(05):77-81.

[15]Liu Xin, Wang Xuyang, Jiang Wei.The influence of Chinese civil servants' work values on job satisfaction -- taking public service motivation as an intermediary variable[J].Chinese administrative management,2016,(12):83-88.

[16]Li Xiaohua, Dong Jun.The impact of public service motivation of civil servants on individual performance[J].Public administration review, 2012, (01): 105-121+181.

[17]Rainey ,H.G.and P.Steinbauer,galloping elephants :developing elements of a theory of effective government organizations .journal of public administration research and theory ,1999(1):1-32.

[18]Ritz,A,public service motivation and organizational performance in Swiss federal government.international review of administrative science ,2009,75(1):p.245-261.

[19]Cai Huajian.Introduction of implicit association test put forward by Greenwald[J].Advances in psychological science,2003,(03):339-344 